\title{
Two Dimensional Laplace Transform Coupled with the Marichev-Saigo-Maeda Integral Operator and the Generalized Incomplete Hypergeometric Function
}

\author{
Yasir Khan ${ }^{1}$, Adnan Khan ${ }^{2}$, Muhammad Shaeel ${ }^{2}$ and Ali Akgül ${ }^{3, *(D)}$ \\ 1 Department of Mathematics, University of Hafr Al Batin, Hafr Al Batin 31991, Saudi Arabia; \\ yasirmath@yahoo.com \\ 2 Department of Mathematics, National College of Business Administration \& Economics, \\ Lahore 54660, Pakistan; adnankhantariq@ncbae.edu.pk (A.K.); muhammad.shaeel123@gmail.com (M.S.) \\ 3 Department of Mathematics, Art and Science Faculty, Siirt University, Siirt 56100, Turkey \\ * Correspondence: aliakgul@siirt.edu.tr
}

check for

updates

Citation: Khan, Y.; Khan, A.; Shaeel, M.; Akgül, A. Two Dimensional Laplace Transform Coupled with the Marichev-Saigo-Maeda Integral Operator and the Generalized Incomplete Hypergeometric Function. Symmetry 2021, 13, 2420. https:/ / doi.org/10.3390/sym13122420

Academic Editor: Calogero Vetro

Received: 10 November 2021 Accepted: 9 December 2021

Published: 14 December 2021

Publisher's Note: MDPI stays neutral with regard to jurisdictional claims in published maps and institutional affiliations.

Copyright: (c) 2021 by the authors. Licensee MDPI, Basel, Switzerland. This article is an open access article distributed under the terms and conditions of the Creative Commons Attribution (CC BY) license (https:// creativecommons.org/licenses/by/ $4.0 /)$.

\begin{abstract}
This paper represents the processing of the two-dimensional Laplace transform with the two-dimensional Marichev-Saigo-Maeda integral operators and two-dimensional incomplete hypergeometric function. This article provides an entirely new perspective on the Marichev-SaigoMaeda operators and incomplete functions. In addition, we have included some interesting results, such as left-sided Saigo-Maeda operators and right-sided Saigo-Maeda operators, making this a good direction for symmetry analysis.
\end{abstract}

Keywords: Laplace transform; integral operators; left-sided Saigo-Maeda operators; right-sided Saigo-Maeda operators

\section{Introduction}

The fractional kinetic equation (FKE) plays a large role in solving astrophysical problems that arise in the field of symmetry analysis. The solutions are represented in terms of the incomplete I-function. In terms of the Laplace function, Khan et al. [1] constructed a Laplace operator using the Caputo fractional differentiation of the extended Mittag-Leffler function. Manzoor et al. [2] developed a Beta operator in terms that was based on the extended Mittag-Leffler function with Caputo fractional differentiation. Bansal et al. [3] used the well-known integral transform to solve the fractional kinetic equation (FKE) associated with the incomplete I-function (IIF) (Laplace transform). The dualities between the Laplace-Carson transform and other useful integral transforms have been described by Chauhan et al. [4]. Agarwal et al. [5,6] applied the generalized Marichev-Saigo-Maeda fractional operator. Saxena et al. [7] developed a new approach to SM functions that contain two-dimensional SM fractional calculus contained with a 2D Aleph transform. Recently Shaeel et al. [8] applied Laplace and inverse Laplace operators on incomplete hypergeometric functions. Srivastava and Saxena [9] provided a systematic (and historical) description of the efforts of various authors into fractional calculus and its applications. The purpose of this paper is to propose a two-dimensional Laplace transformation that is linked to the Marichev-Saigo-Maeda Integral Operator and the generalized incomplete hypergeometric function. Furthermore, we discussed the special cases and discovered several interesting corollaries. Compared to the aforementioned existing operators, this work would be an excellent addition to integral operators. 


\section{Preliminaries}

Definition 1. $\gamma(p ; X)$ and $\Gamma[p ; X]$ are incomplete gamma functions that are defined as

$$
\gamma(p ; X)=\int_{0}^{X} t^{p-1} e^{-t} d t,(\Re(p)>0, X \geq 0),
$$

and

$$
\Gamma[p ; X]=\int_{X}^{\infty} t^{p-1} e^{-t} d t,(\Re(p)>0, X \geq 0) .
$$

Definition 2. The gamma decomposition formula is

$$
\gamma(p ; X)+\Gamma[p ; X]=\Gamma(p),(\Re(p)>0, X \geq 0) .
$$

Here, $\Gamma(p)$ is a gamma function.

\section{Definition 3.}

$$
\begin{gathered}
\Gamma(p)=\int_{0}^{\infty} X^{p-1} e^{-X} d X,(\Re(p)>0), \\
(\alpha: X)_{n}=\frac{\gamma(\alpha+n X)}{\gamma(X)},
\end{gathered}
$$

and

$$
[\alpha: X]_{n}=\frac{\Gamma(\alpha+n X)}{\Gamma(X)} .
$$

Definition 4. The decomposed formula for the Pochhammer symbol $(\alpha)_{n}[10]$ is as follows:

$$
(\alpha: X)_{n}+[\alpha: X]_{n}=(\alpha)_{n},
$$

where

$$
\begin{gathered}
(\alpha)_{n}=\prod_{t=1}^{n}(\alpha+t-1) \\
(\alpha)_{n}=(\alpha)(\alpha+1)(\alpha+2) \ldots(\alpha+n-1) \\
(\alpha)_{0}=1, \alpha \neq 0 .
\end{gathered}
$$

Definition 5. The incomplete hypergeometric function was created by Srivastava et al. [11]

$$
p \gamma_{q}\left[\begin{array}{c}
\left(\alpha_{1} ; X\right),\left(\alpha_{2}\right),\left(\alpha_{3}\right), \ldots,\left(\alpha_{p}\right) ; z \\
\left(b_{1}\right),\left(b_{2}\right), \ldots,\left(b_{q}\right) ;
\end{array}\right]=\left\{\sum_{n=0}^{x} \frac{\left(\alpha_{1} ; X\right),\left(\alpha_{2}\right), \ldots,\left(\alpha_{p}\right)}{\left(b_{1}\right), \ldots,\left(b_{q}\right)} \frac{z^{n}}{n !}\right\},
$$

and

$$
{ }_{p} \Gamma_{q}\left[\begin{array}{c}
{\left[a_{1} ; X\right],\left(a_{2}\right),\left(a_{3}\right), \ldots,\left(a_{p}\right) ;} \\
\left(b_{1}\right),\left(b_{2}\right), \ldots,\left(b_{q}\right) ;
\end{array}\right]=\left\{\sum_{n=x}^{\infty} \frac{\left[a_{1} ; X\right],\left(a_{2}\right), \ldots,\left(a_{p}\right)}{\left(b_{1}\right), \ldots,\left(b_{q}\right)} \frac{z^{n}}{n !}\right\},
$$

where the variables $\left(a_{1} ; X\right)_{n}$ and $\left[a_{1} ; X\right]_{n}$ are presented in $[0, X]$ and $[X, \infty)$. Therefore, we have ${ }_{p} F_{q} ;\left(p ; q \in N_{0}\right)$ 


$$
\begin{gathered}
p \gamma_{q}\left[\begin{array}{c}
\left(\alpha_{1} ; X\right),\left(\alpha_{2}\right),\left(\alpha_{3}\right), \ldots,\left(\alpha_{p}\right) ; \\
\left(b_{1}\right),\left(b_{2}\right), \ldots,\left(b_{q}\right) ;
\end{array}\right]+{ }_{p} \Gamma_{q}\left[\begin{array}{c}
{\left[\alpha_{1} ; X\right],\left(\alpha_{2}\right),\left(\alpha_{3}\right), \ldots,\left(\alpha_{p}\right) ;} \\
\left(b_{1}\right),\left(b_{2}\right), \ldots,\left(b_{q}\right) ;
\end{array}\right]= \\
{ }_{p} F_{q}\left[\begin{array}{c}
\left(\alpha_{1}\right),\left(\alpha_{2}\right),\left(\alpha_{3}\right), \ldots,\left(\alpha_{p}\right) ; \\
\left(b_{1}\right),\left(b_{2}\right), \ldots,\left(b_{q}\right) ;
\end{array}\right] .
\end{gathered}
$$

\section{Definition 6.}

$$
{ }_{2} F_{1}(a, b ; c ; x)=\sum_{m=0}^{\infty} \frac{(a)_{m}(b)_{m}}{(c)_{m}} \cdot \frac{x^{m}}{m !} .
$$

Appell function by Rainville [10] of the first kind $F_{3}$ is a two-variable hypergeometric function that is described by:

$$
\begin{gathered}
F_{3}\left(\varphi, \varphi^{\prime}, v, v^{\prime}, \eta ; s, p\right)=\sum_{m, n}^{\infty} \frac{(\varphi)_{m}\left(\varphi^{\prime}\right)_{n}(v)_{m}\left(v^{\prime}\right)_{n}}{(\eta)_{m+n}} \frac{s^{m}}{m !} \frac{p^{n}}{n !} \\
=\sum_{m=0}^{\infty} \frac{(\varphi)_{m}(v)_{m}}{(\eta)_{m}}{ }_{2} F_{1}\left[\begin{array}{cc}
\varphi^{\prime}, v^{\prime} \\
\eta+m
\end{array} \quad y\right] \frac{s^{m}}{m !} .
\end{gathered}
$$

Definition 7. The left-sided generalized fractional integration operators involving Appell function $F_{3}$ are described by Saigo and Maeda [12] through the following equation:

Let $\omega, \omega^{\prime}, v, v^{\prime}, \eta \in C, x>0,(\Re(\eta)>0)$

$$
\begin{gathered}
\left(I_{0+}{ }^{\omega, \omega^{\prime}, v, v^{\prime}, \eta} f\right)(x)=\frac{x^{-\omega}}{\Gamma(\eta)} \int_{0}^{x} t^{-\omega^{\prime}}(x-t)^{\eta-1} \\
\times F_{3}\left(\omega, \omega^{\prime}, v, v^{\prime}, \eta ; 1-\frac{t}{x}, 1-\frac{x}{t}\right) f(t) d t \\
=\frac{d^{n}}{d x^{n}}\left(I_{0+}^{\omega, \omega^{\prime}, v+\omega, v^{\prime}, \eta+\omega} f\right)(x)
\end{gathered}
$$

where $\Re(\eta) \leq 0$ and $n=[\Re(-\eta)+1]$.

Definition 8. Nadir and Khan [13] considered the composition of the left-sided MSM fractional integration as shown

$$
\begin{aligned}
& \left(I_{0+} \omega^{\prime} \omega^{\prime}, v, v^{\prime}, \eta t^{\alpha-1}\right)(x)=\frac{\Gamma(\alpha) \Gamma\left(\alpha+\eta-\omega-\omega^{\prime}-v\right) \Gamma\left(\alpha+v^{\prime}-\omega^{\prime}\right)}{\Gamma\left(\alpha+\eta-\omega-\omega^{\prime}\right) \Gamma\left(\alpha+\eta-\omega^{\prime}-v\right) \Gamma\left(\alpha+v^{\prime}\right)} \times x^{\alpha-\omega-\omega^{\prime}+\eta-1} \\
& \text { where }\left(\Re(\eta)>0, \Re(\alpha)>\max \left[0, \Re\left(\omega+\omega^{\prime}+v-\eta\right), \Re\left(\omega^{\prime}-v^{\prime}\right)\right]\right) \text {. }
\end{aligned}
$$

Definition 9. The right-sided generalized fractional integration operators containing the Appell function $F_{3}$ are described by Saigo and Maeda [12] by the following equation:

Let $\omega, \omega^{\prime}, v, v^{\prime}, \eta \in C, x>0,(\Re(\eta)>0)$

$$
\begin{gathered}
\left(I_{0-}^{\omega, \omega^{\prime}, v, v^{\prime}, \eta} f\right)(x)=\frac{x^{-\omega^{\prime}}}{\Gamma(\eta)} \int_{x}^{\infty} t^{-\omega}(t-x)^{\eta-1} \\
\times F_{3}\left(\omega, \omega^{\prime}, v, v^{\prime}, \eta ; 1-\frac{t}{x}, 1-\frac{x}{t}\right) f(t) d t \\
=(-1)^{n} \frac{d^{n}}{d x^{n}}\left(I_{0-}^{\omega, \omega^{\prime}, v, v^{\prime}+\omega, \eta+\omega} f\right)(x), \\
(\Re(\eta)>0 ; \mathrm{n}=[\Re(\eta)+1]) .
\end{gathered}
$$

Definition 10. Nadir and Khan [13] considered the composition of the right-sided MSM fractional integration as shown 


$$
\left(I_{0-}{ }^{\omega} \omega^{\prime}, v, v^{\prime}, \eta t^{\alpha-1}\right)(x)=\frac{\Gamma\left(1+\omega+\omega^{\prime}-\eta-\alpha\right) \Gamma\left(1+\omega+v^{\prime}-\eta-\alpha\right) \Gamma(1-v-\alpha)}{\Gamma(1-\alpha) \Gamma\left(1+\omega+\omega^{\prime}+v^{\prime}-\eta-\alpha\right) \Gamma(1+\omega-v-\alpha)} \times x^{\alpha-\omega-\omega^{\prime}+\eta-1},
$$

where

$$
\left(\Re(\eta)>0, \Re(\alpha)>1+\min \left[\Re(-v), \Re\left(\omega+\omega^{\prime}-\eta\right), \Re\left(\omega^{\prime}-v^{\prime}-\eta\right)\right]\right)
$$

Definition 11. The left-sided Saigo fractional integration operator including Gaussian hypergeometric function ${ }_{2} F_{1}$ by Saigo [14] can be depicted as

Let $\omega, v, \eta, \alpha \in C$ and $x>0, \Re(\eta)>0$

$$
\begin{gathered}
\left(I_{0+}^{\omega, v, \eta} f\right)(x)=\frac{x^{-\omega-v}}{\Gamma(\omega)} \int_{0}^{x}(x-t)^{\omega-1} t^{-v-\omega} \\
\times{ }_{2} F_{1}\left(\omega+v,-\eta ; \omega ; 1-\frac{x}{t}\right) f(t) d t \\
=\frac{d^{n}}{d x^{n}}\left(I_{0+}^{\omega+n, v-n, \eta-n} f\right)(x),
\end{gathered}
$$

where $\Re(\eta) \leq 0$ and $n=[\Re(-\eta)+1]$.

Definition 12. The left-sided Saigo integration operator as used by Saxena et al. [13] can be defined as

$$
\left(I_{0+}^{\omega, v, \eta} t^{\alpha-1}\right)(x)=\frac{\Gamma(\alpha) \Gamma(\alpha+\eta-v)}{\Gamma(\alpha-v) \Gamma(\alpha+\omega+\eta)} x^{\alpha-v-1},
$$

where $\Re(\alpha)>\max \{0, \Re(v-\eta)\}$.

Definition 13. The right-sided Saigo fractional integration operator containing Gaussian hypergeometric function by Saigo [8] can be defined as

Let $\omega, v, \eta, \alpha \in C$ and $x>0, \Re(\eta)>0$

$$
\begin{gathered}
\left(I_{0-}^{\omega, v, \eta} f\right)(x)=\frac{1}{\Gamma(\omega)} \int_{x}^{\infty}(t-x)^{\omega-1} t^{-\omega-v} \\
\times{ }_{2} F_{1}\left(\omega+v,-\eta ; \omega ;, 1-\frac{x}{t}\right) f(t) d t \\
=(-1) \frac{d^{n}}{d x^{n}}\left(I_{0-}^{\omega-n, v+n, \eta} f\right)(x),
\end{gathered}
$$

where $\Re(\eta) \leq 0$ and $n=[\Re(-\eta)+1]$.

Definition 14. The right-sided Saigo integration operator as used by Saxena et al. [13] can be depicted as

$$
\left(I_{0-}^{\omega, v, \eta} t^{\alpha-1}\right)(x)=\frac{\Gamma(1+v-\alpha) \Gamma(1+\eta-\alpha)}{\Gamma(1-\alpha) \Gamma(1-\alpha+\omega+v+\eta)} x^{\alpha-v-1},
$$

where $\Re(\alpha)<1+\min \{\Re(v), \Re(\eta)\}$ and $x>0$.

Definition 15. Following Saxena et al. [13], we assign $h_{1}$ the class of function $f(x)$ on $\Re+$ when $x$ tends to $\infty$ for all $\Omega$. Similarly, by $h_{2}$ the class of the functions $f(x, y)$ of $\Re+\times \Re+$ when $x$ and $y$ both tend to $\infty$ for all $\Omega_{i}(i=1,2)$. The two-dimensional Saigo-Maeda operator of a Weyl-type of order $\Re(\eta)>0, \Re(\tau)>0$ is shown in the different kinds of functions $f(x, y)$. 


$$
\begin{gathered}
\left(I_{x+}{ }^{\omega, \omega^{\prime}, v, v^{\prime}, \eta}\right)\left(I_{y+}^{\varphi, \varphi^{\prime}, \delta, \delta^{\prime}, \tau}\right) f(x, y)= \\
x^{\omega+\omega^{\prime}-\eta} y^{\varphi+\varphi^{\prime}-\tau} \frac{x^{-\omega^{\prime}}}{\Gamma(\eta)} \frac{y^{-\varphi^{\prime}}}{\Gamma(\tau)} \int_{x}^{\infty} \int_{y}^{\infty} t^{-\omega} u^{-\varphi}(t-x)^{\eta-1}(u-y)^{\tau-1} \times \\
F_{3}\left(\omega, \omega^{\prime}, v, v^{\prime}, \eta ; 1-\frac{t}{x}, 1-\frac{x}{t}\right) \\
\times F\left(\varphi, \varphi^{\prime}, \delta, \delta^{\prime}, \tau ; 1-\frac{u}{y}, 1-\frac{y}{u}\right) f(t, u) d t d u .
\end{gathered}
$$

Definition 16. The Laplace transform of a function $f(x, y) \in h_{2}$ is defined as

$$
L[f(z, r) ; w, k]=\int_{0}^{\infty} \int_{0}^{\infty} e^{-w z-k r} f(z, r) d z d r,
$$

$(\Re(\mathrm{z})>0, \Re(\mathrm{r})>0)$.

Here, we assume that $L[f(z, r) ; w, k]$ exists and belongs to $h_{2}$.

3. Two-Dimensional Laplace Transform Coupled with the Left-Sided MSM Integral Operator and the Generalized Incomplete Hypergeometric Function

Theorem 1. Assume $\omega, \omega^{\prime}, v, v^{\prime}, \eta ; \varphi, \varphi^{\prime}, \delta, \delta^{\prime}, \tau \in \mathrm{C}$, such that $\Re(\eta)>0, \Re(\tau)>0$; if conditions $\quad \Re(\eta) \quad>\quad \max \left[0, \Re\left(\eta-\omega-\omega^{\prime}-v^{\prime}\right),(v-\omega)\right] \quad$ and $\Re(\tau)>\max \left[0, \Re\left(\tau-\varphi-\varphi^{\prime}-\delta^{\prime}\right),(\delta-\varphi)\right]$ both are hold. Therefore, the left-sided SaigoMaeda operator for the integration of an incomplete hypergeometric function is presented as:

$$
\begin{aligned}
& L\left[\begin{array}{c}
z^{i-1} r^{0-1} ; z, r \times\left(I_{x+} \omega_{, \omega^{\prime}, v, v^{\prime}, \eta}\left(t^{\alpha-1}{ }_{\rho} \gamma_{q}(z t)\right)\right) \\
\left(I_{y+}^{\varphi, \varphi^{\prime}, \delta, \delta^{\prime}, \tau}\left(G^{\beta-1}{ }_{\rho^{\prime}} \gamma^{\prime}{ }^{\prime}(r g)\right)\right)
\end{array}\right](x, y)= \\
& \Gamma(i) \Gamma(o) \Gamma(\alpha) \Gamma(\beta) \Gamma\left(\alpha+\eta-\omega-\omega^{\prime}-v\right) \\
& \frac{1}{w^{i} k^{0}} x^{\alpha-\omega-\omega^{\prime}+\eta-1} x^{\beta-\varphi-\varphi^{\prime}+\tau-1} \frac{\Gamma\left(\beta+\tau-\varphi-\varphi^{\prime}-\delta\right) \Gamma\left(\alpha+v^{\prime}-\omega^{\prime}\right) \Gamma\left(\beta+\delta^{\prime}-\varphi^{\prime}\right)}{\Gamma\left(\alpha+\eta-\omega-\omega^{\prime}\right) \Gamma\left(\beta+\tau-\varphi-\varphi^{\prime}\right) \Gamma\left(\alpha+\eta-\omega^{\prime}-v\right)} \\
& \Gamma\left(\beta+\tau-\varphi^{\prime}-\delta\right) \Gamma\left(\alpha+v^{\prime}\right) \Gamma\left(\beta+\delta^{\prime}\right)
\end{aligned}
$$

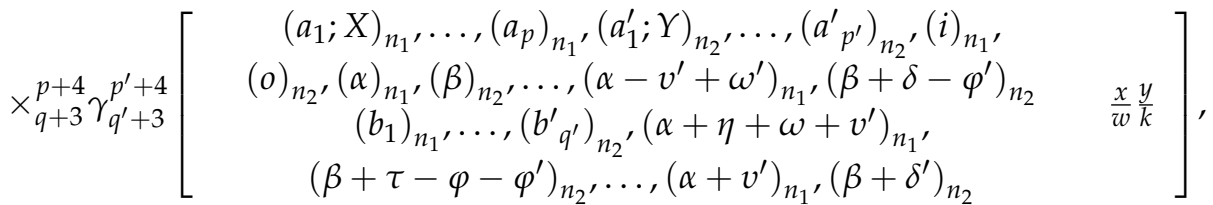




$$
\begin{aligned}
& \text { and } \\
& L\left[\begin{array}{c}
z^{i-1} r^{o-1} ; z, r \times\left(I_{x+} \omega_{, \omega^{\prime}, v, v^{\prime}, \eta}\left(t^{\alpha-1}{ }_{\rho} \Gamma_{q}(z t)\right)\right) \\
\left(I_{y+}^{\varphi, \varphi^{\prime}, \delta, \delta^{\prime}, \tau}\left(G^{\beta-1}{ }_{\rho^{\prime}} \Gamma_{\prime_{q}}(r g)\right)\right)
\end{array}\right](x, y)= \\
& \Gamma(i) \Gamma(o) \Gamma(\alpha) \Gamma(\beta) \Gamma\left(\alpha+\eta-\omega-\omega^{\prime}-v+\right) \\
& \frac{1}{w^{i} k^{0}} x^{\alpha-\omega-\omega^{\prime}+\eta-1} x^{\beta-\varphi-\varphi^{\prime}+\tau-1} \frac{\Gamma\left(\beta+\tau-\varphi-\varphi^{\prime}-\delta\right) \Gamma\left(\alpha+v^{\prime}-\omega^{\prime}\right) \Gamma\left(\beta+\delta^{\prime}-\varphi^{\prime}\right)}{\Gamma\left(\alpha+\eta-\omega-\omega^{\prime}\right) \Gamma\left(\beta+\tau-\varphi-\varphi^{\prime}+n_{2}\right) \Gamma\left(\alpha+\eta-\omega^{\prime}-v\right)} \\
& \Gamma\left(\beta+\tau-\varphi^{\prime}-\delta\right) \Gamma\left(\alpha+v^{\prime}\right) \Gamma\left(\beta+\delta^{\prime}\right) \\
& \times_{q+3}^{p+4} \Gamma_{q^{\prime}+3}^{p^{\prime}+4}\left[\begin{array}{cc}
\left(a_{1} ; X\right)_{n_{1}}, \ldots,\left(a_{p}\right)_{n_{1}}\left(a_{1}^{\prime} ; Y\right)_{n_{2}}, \ldots,\left(a_{p^{\prime}}^{\prime}\right)_{n_{2}{ }^{\prime}}(i)_{n_{1^{\prime}}} & \\
(o)_{n_{2}}(\alpha)_{n_{1}},(\beta)_{n_{2}} \ldots,\left(\alpha-v^{\prime}+\omega^{\prime}\right)_{n_{1}},\left(\beta+\delta-\varphi^{\prime}\right)_{n_{2}} & \frac{x}{w} \frac{y}{k} \\
\left(b_{1}\right)_{n_{1}}, \ldots,\left(b^{\prime}{ }_{q^{\prime}}\right)_{n_{2}}\left(\alpha+\eta+\omega+v^{\prime}\right)_{n_{1}} & \\
\left(\beta+\tau-\varphi-\varphi^{\prime}\right)_{n_{2}}, \ldots,\left(\alpha+v^{\prime}\right)_{n_{1}}\left(\beta+\delta^{\prime}\right)_{n_{2}} &
\end{array}\right] .
\end{aligned}
$$

Proof. Let us take the incomplete hypergeometric function (11) and proceed with the MSM integration operator (19) and two-dimensional Laplace transform; by interchanging the summation, we obtain the required result.

$$
\begin{aligned}
& L\left[\begin{array}{c}
z^{i-1} r^{o-1} ; z, r \times\left(I_{x+} \omega_{, \omega^{\prime}, v, v^{\prime}, \eta}\left(t^{\alpha-1} \gamma_{\rho}(z t)\right)\right) \\
\left(I_{y+}^{\varphi, \varphi^{\prime}, \delta, \delta^{\prime}, \tau}\left(G_{\rho^{\prime}}^{\beta-1} \gamma_{\prime}(r g)\right)\right)
\end{array}\right](x, y)= \\
& L\left[z^{i-1} r^{o-1} ; z, r\right]\left\{\begin{array}{c}
\sum_{n_{1}=0}^{x} \frac{\left(a_{1} ; X\right)_{n_{1}}\left(a_{2}\right)_{n_{1}}, \ldots,\left(a_{p}\right)_{n_{1}}}{\left(b_{1}\right)_{n_{1}}\left(b_{2}\right)_{n_{1}}, \ldots,\left(b_{q}\right)_{n_{1}}} \frac{z^{n_{1}}}{n_{1} !} \times \\
\sum_{n_{2}=0}^{y} \frac{\left(a_{1}^{\prime} ;\right)_{n_{2}}\left(a_{2}^{\prime}\right)_{n_{2}, \ldots,\left(a^{\prime}{ }^{\prime}\right)_{n}}}{\left(b_{1}^{\prime}\right)_{n_{2}}\left(b_{2}^{\prime}\right)_{n_{2}}, \ldots,\left(b^{\prime} q_{q^{\prime}}\right)_{n_{2}}} \frac{z^{n_{2}}}{n_{2} !}
\end{array}\right\} \times \\
& \left(I_{x+}{ }^{\omega, \omega^{\prime}, v, v^{\prime}, \eta}\left(t^{\alpha+n_{1}-1}\right)\right)(x) \times\left(I_{y+}^{\varphi, \varphi^{\prime}, \delta, \delta^{\prime}, \tau}\left(g^{\beta+n_{2}-1}\right)\right)(y) \\
& L\left[\begin{array}{c}
z^{i-1} r^{o-1} ; z, r \times\left(I_{x+}{ }^{\omega, \omega^{\prime}, v, v^{\prime}, \eta}\left(t^{\alpha-1}{ }_{\rho} \gamma_{q}(z t)\right)\right) \\
\left(I_{y+}^{\varphi, \varphi^{\prime}, \delta, \delta^{\prime}, \tau}\left(G^{\beta-1}{ }_{\rho^{\prime}} \gamma_{\prime}(r g)\right)\right)
\end{array}\right](x, y)= \\
& \times\left\{\begin{array}{c}
\sum_{n_{1}=0}^{x} \frac{\left(a_{1} ; X\right)_{n_{1}},\left(a_{2}\right)_{n_{1}}, \ldots,\left(a_{p}\right)_{n_{1}}}{\left(b_{1}\right)_{n_{1}}\left(b_{2}\right)_{n_{1}}, \ldots,\left(b_{q}\right)_{n_{1}}} \frac{1}{n_{1} !} \times \\
\sum_{n_{2}=0}^{y} \frac{\left(a_{1}^{\prime} ;\right)_{n_{2}}\left(a_{2}^{\prime}\right)_{n_{2}}, \ldots,\left(a^{\prime} p^{\prime}\right)_{n_{2}}}{\left.\left(b_{1}^{\prime}\right)_{n_{2}}\left(b_{2}^{\prime}\right)_{n_{2}}, \ldots, b^{\prime} q_{q^{\prime}}\right)_{n_{2}}} \frac{1}{n_{2} !}
\end{array}\right\} \\
& \times \frac{\Gamma\left(\alpha+n_{1}\right) \Gamma\left(\alpha+\eta-\omega-\omega^{\prime}-v+n_{1}\right)}{\Gamma\left(\alpha+v^{\prime}-\omega^{\prime}+n_{1}\right)} x^{\alpha-\omega-\omega^{\prime}+\eta+n_{1}-1} \\
& \Gamma\left(\beta+n_{2}\right) \Gamma\left(\beta+\tau-\varphi-\varphi^{\prime}-\delta+n_{2}\right) \\
& \times \frac{\Gamma\left(\beta+\delta^{\prime}-\varphi^{\prime}+n_{2}\right)}{\Gamma\left(\beta+\tau-\varphi-\varphi^{\prime}+n_{2}\right)} x^{\beta-\varphi-\varphi^{\prime}+\tau+n_{2}-1} \\
& \times \int_{0}^{\infty} \int_{0}^{\infty} e^{-z w-r k} z^{i+n_{1}-1} r^{o+n_{2}-1} d z d r .
\end{aligned}
$$




$$
\begin{aligned}
& L\left[z^{i-1} r^{o-1} ; z, r \times\left(I_{x+}{ }^{\omega, \omega^{\prime}, v, v^{\prime}, \eta}\left(t_{\rho-1}^{\alpha} \gamma_{q}(z t)\right)\right)\left(I_{y+}^{\varphi, \varphi^{\prime}, \delta, \delta^{\prime}, \tau}\left(G_{\rho^{\prime}}^{\beta-1} \gamma_{\prime q}(r g)\right)\right)\right](x, y)= \\
& \left\{\sum_{n_{1}=0}^{x} \frac{\left(a_{1} ; X\right)_{n_{1}}\left(a_{2}\right)_{n_{1}}, \ldots,\left(a_{p}\right)_{n_{1}}}{\left(b_{1}\right)_{n_{1}}\left(b_{2}\right)_{n_{1}, \ldots,},\left(b_{q}\right)_{n_{1}}} \frac{1}{n_{1} !} \times \sum_{n_{2}=0}^{y} \frac{\left(a_{1}^{\prime} ; Y\right)_{n_{2}},\left(a_{2}^{\prime}\right)_{n_{2}}, \ldots,\left(a^{\prime}{ }_{p^{\prime}}\right)_{n_{2}}}{\left(b_{1}^{\prime}\right)_{n_{2}}\left(b_{2}^{\prime}\right)_{n_{2}}, \ldots,\left(b^{\prime}{ }_{q^{\prime}}\right)_{n_{2}}} \frac{1}{n_{2} !}\right\} \\
& \times \frac{\Gamma\left(\alpha+n_{1}\right) \Gamma\left(\alpha+\eta-\omega-\omega^{\prime}-v+n_{1}\right) \Gamma\left(\alpha+v^{\prime}-\omega^{\prime}+n_{1}\right)}{\Gamma\left(\alpha+\eta-\omega-\omega^{\prime}+n_{1}\right) \Gamma\left(\alpha+\eta-\omega^{\prime}-v+n_{1}\right) \Gamma\left(\alpha+v^{\prime}+n_{1}\right)} x^{\alpha-\omega-\omega^{\prime}+\eta+n_{1}-1} \\
& \times \frac{\Gamma\left(\beta+n_{2}\right) \Gamma\left(\beta+\tau-\varphi-\varphi^{\prime}-\delta+n_{2}\right) \Gamma\left(\beta+\delta^{\prime}-\varphi^{\prime}+n_{2}\right)}{\Gamma\left(\beta+\tau-\varphi-\varphi^{\prime}+n_{2}\right) \Gamma\left(\beta+\tau-\varphi^{\prime}-\delta+n_{2}\right) \Gamma\left(\beta+\delta^{\prime}+n_{2}\right)} x^{\beta-\varphi-\varphi^{\prime}+\tau+n_{2}-1} .
\end{aligned}
$$

$$
\begin{aligned}
& L\left[\begin{array}{c}
z^{i-1} r^{o-1} ; z, r \times\left(I_{x+}{ }^{\omega, \omega^{\prime}, v, v^{\prime}, \eta}\left(t^{\alpha-1}{ }_{\rho} \gamma_{q}(z t)\right)\right) \\
\left(I_{y+}^{\varphi, \varphi^{\prime}, \delta, \delta^{\prime}, \tau}\left(G^{\beta-1}{ }_{\rho^{\prime}} \gamma_{\prime}^{\prime}(r g)\right)\right)
\end{array}\right](x, y)= \\
& \left\{\begin{array}{c}
\sum_{n_{1}=0}^{x} \frac{\left(a_{1} ; X\right)_{n_{1}},\left(a_{2}\right)_{n_{1}}, \ldots,\left(a_{p}\right)_{n_{1}}}{\left(b_{1}\right)_{n_{1}}\left(b_{2}\right)_{n_{1}}, \ldots,\left(b_{q}\right)_{n_{1}}} \frac{1}{n_{1} !} \times \\
\sum_{n_{2}=0}^{y} \frac{\left(a_{1}^{\prime} ; Y\right)_{n_{2}}\left(a_{2}^{\prime}\right)_{n_{2}}, \ldots,\left(a^{\prime}{ }_{p^{\prime}}\right)_{n_{2}}}{\left(b_{1}^{\prime}\right)_{n_{2}}\left(b_{2}^{\prime}\right)_{n_{2}}, \ldots,\left(b^{\prime}{ }_{q^{\prime}}\right)_{n_{2}}} \frac{1}{n_{2} !}
\end{array}\right\} \times \\
& \Gamma\left(\alpha+n_{1}\right) \Gamma\left(\alpha+\eta-\omega-\omega^{\prime}-v+n_{1}\right) \\
& \frac{\Gamma\left(\alpha+v^{\prime}-\omega^{\prime}+n_{1}\right)}{\Gamma\left(\alpha+\eta-\omega-\omega^{\prime}+n_{1}\right)} x^{\alpha-\omega-\omega^{\prime}+\eta+n_{1}-1} \times \\
& \Gamma\left(\alpha+\eta-\omega^{\prime}-v+n_{1}\right) \Gamma\left(\alpha+v^{\prime}+n_{1}\right) \\
& \Gamma\left(\beta+n_{2}\right) \Gamma\left(\beta+\tau-\varphi-\varphi^{\prime}-\delta+n_{2}\right) \\
& \begin{array}{l}
\frac{\Gamma\left(\beta+\delta^{\prime}-\varphi^{\prime}+n_{2}\right)}{\Gamma\left(\beta+\tau-\varphi-\varphi^{\prime}+n_{2}\right)} x^{\beta-\varphi-\varphi^{\prime}+\tau+n_{2}-1} \frac{(i)_{n_{1}} \Gamma(i)}{w^{i}} \frac{(o)_{n_{2}} \Gamma(o)}{k^{o}} . \\
\Gamma\left(\beta+\tau-\varphi^{\prime}-\delta+n_{2}\right) \Gamma\left(\beta+\delta^{\prime}+n_{2}\right)
\end{array}
\end{aligned}
$$

Therefore, we obtain the desired result. For the second assertion, it takes an incomplete hypergeometric function (12) and the same results are obtained.

\section{Two-Dimensional Laplace Transform Coupled with the Right-Sided MSM Integral Operator and Generalized Incomplete Hypergeometric Function}

Theorem 2. Assume $\omega, \omega^{\prime}, v, v^{\prime}, \eta ; \varphi, \varphi^{\prime}, \delta, \delta^{\prime}, \tau \in \mathrm{C}$ and $\left|\frac{1}{t}\right| \leq 1,\left|\frac{1}{g}\right| \leq 1$ such that $(\Re(\eta)>$ $0, \Re(\tau)>0)$ if the condition $\Re(\eta)>-\min \left[0, \Re\left(\eta-\omega-\omega^{\prime}-v^{\prime}\right),(v-\omega)\right]$ and $\Re(\tau)>$ $-\min \left[0, \Re\left(\tau-\varphi-\varphi^{\prime}-\delta^{\prime}\right),(\delta-\varphi)\right]$ hold; thus the left-sided The Saigo-Maeda operator of differentiation of the incomplete hypergeometric function is presented as: 


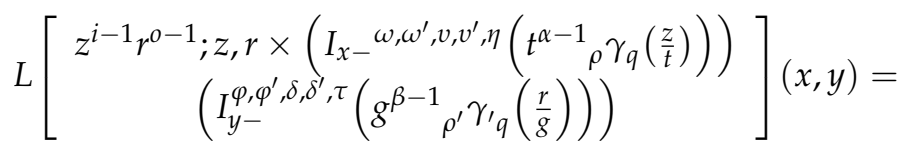

$$
\begin{aligned}
& \frac{1}{w^{i} k^{0}} x^{\alpha-\omega-\omega^{\prime}+\eta-1} x^{\beta-\varphi-\varphi^{\prime}+\tau-1} \\
& \Gamma(i) \Gamma(o) \Gamma\left(1+\omega+\omega^{\prime}-\alpha-\eta\right) \Gamma\left(1+\varphi+\varphi^{\prime}-\beta-\tau\right) \\
& \frac{\Gamma\left(1+\omega+v^{\prime}-\eta-\alpha\right) \Gamma\left(1+\varphi+\delta^{\prime}-\beta-\tau\right) \Gamma(1-v-\alpha) \Gamma(1-\delta-\beta)}{\Gamma(1-\alpha) \Gamma(1-\beta) \Gamma\left(1+\omega+\omega^{\prime}+v^{\prime}-\alpha-\eta\right)} \\
& \Gamma\left(1+\varphi+\varphi^{\prime}+\delta^{\prime}-\beta-\tau\right) \Gamma(1+\omega-v-\alpha) \Gamma(1+\varphi-\delta-\beta)
\end{aligned}
$$

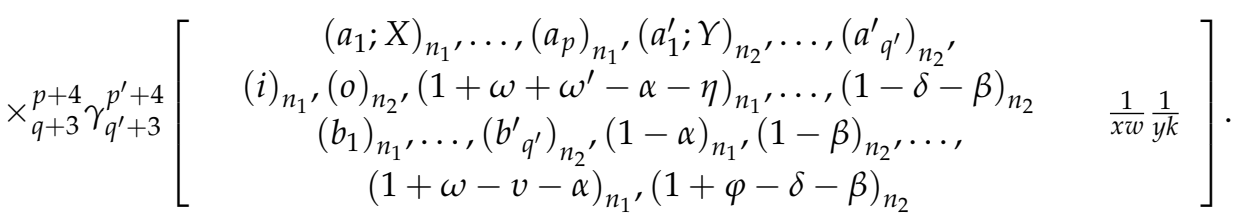

and

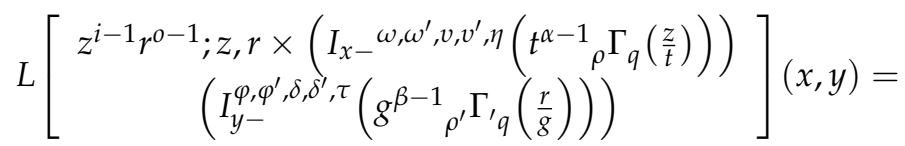

$$
\begin{aligned}
& \frac{1}{w^{i} k^{0}} x^{\alpha-\omega-\omega^{\prime}+\eta-1} x^{\beta-\varphi-\varphi^{\prime}+\tau-1} \\
& \Gamma(i) \Gamma(o) \Gamma\left(1+\omega+\omega^{\prime}-\alpha-\eta\right) \Gamma\left(1+\varphi+\varphi^{\prime}-\beta-\tau\right) \\
& \frac{\Gamma\left(1+\omega+v^{\prime}-\eta-\alpha\right) \Gamma\left(1+\varphi+\delta^{\prime}-\beta-\tau\right) \Gamma(1-v-\alpha) \Gamma(1-\delta-\beta)}{\Gamma(1-\alpha) \Gamma(1-\beta) \Gamma\left(1+\omega+\omega^{\prime}+v^{\prime}-\alpha-\eta\right)} \\
& \Gamma\left(1+\varphi+\varphi^{\prime}+\delta^{\prime}-\beta-\tau\right) \Gamma(1+\omega-v-\alpha) \Gamma(1+\varphi-\delta-\beta)
\end{aligned}
$$

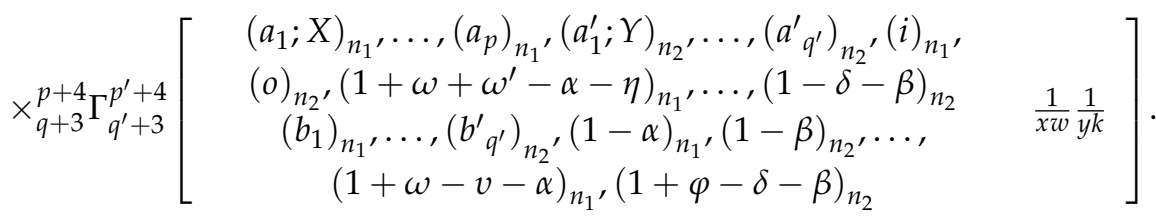

Proof. Let us take the incomplete hypergeometric function (11) to proceed with the MSM integration operator (22) and two-dimensional Laplace transform; by interchanging the summation, we obtain the required result.

$$
\begin{aligned}
& L\left[\begin{array}{c}
z^{i-1} r^{o-1} ; z, r \times\left(I_{x-} \omega, \omega^{\prime}, v, v^{\prime}, \eta\left(t^{\alpha-1}{ }_{\rho} \gamma_{q}\left(\frac{z}{t}\right)\right)\right) \\
\left(I_{y-}^{\varphi, \varphi^{\prime}, \delta, \delta^{\prime}, \tau}\left(g^{\beta-1}{ }_{\rho^{\prime}} \gamma_{\prime_{q}}\left(\frac{r}{g}\right)\right)\right)
\end{array}\right](x, y)= \\
& L\left[z^{i-1} r^{o-1} ; z, r\right]\left\{\begin{array}{c}
\sum_{n_{1}=0}^{x} \frac{\left(a_{1} ; X\right)_{n_{1}}\left(a_{2}\right)_{n_{1}}, \ldots,\left(a_{p}\right)_{n_{1}}}{\left(b_{1}\right)_{n_{1}}\left(b_{2}\right)_{n_{1}}, \ldots,\left(b_{q}\right)_{n_{1}}} \frac{z^{n_{1}}}{n_{1} !} \times \\
\sum_{n_{2}=0}^{y} \frac{\left(a_{1}^{\prime} ; Y\right)_{n_{2}}\left(a_{2}^{\prime}\right)_{n_{2}}, \ldots,\left(a^{\prime}{ }^{\prime}\right)_{n_{2}}}{\left.\left(b_{1}^{\prime}\right)_{n_{2}}{ }^{\prime}\left(b_{2}^{\prime}\right)_{n_{2}}, \ldots, b^{\prime}{ }_{q^{\prime}}\right)_{n_{2}}} \frac{z^{n_{2}}}{n_{2} !}
\end{array}\right\} \times \\
& \left(I_{x-} \omega \omega^{\prime}, v, v^{\prime}, \eta\left(t^{\alpha-n_{1}-1}\right)\right)(x) \times\left(I_{y-}^{\varphi, \varphi^{\prime}, \delta, \delta^{\prime}, \tau}\left(g^{\beta-n_{2}-1}\right)\right)(y) .
\end{aligned}
$$




$$
\begin{aligned}
& L\left[\begin{array}{c}
z^{i-1} r^{o-1} ; z, r \times\left(I_{x-} \omega, \omega^{\prime}, v, v^{\prime}, \eta\left(t^{\alpha-1}{ }_{\rho} \gamma_{q}\left(\frac{z}{t}\right)\right)\right) \\
\left(I_{y-}^{\varphi, \varphi^{\prime}, \delta, \delta^{\prime}, \tau}\left(g^{\beta-1}{ }_{\rho^{\prime}} \gamma_{\prime_{q}}\left(\frac{r}{g}\right)\right)\right)
\end{array}\right](x, y)= \\
& \left\{\begin{array}{c}
\sum_{n_{1}=0}^{x} \frac{\left(a_{1} ; X\right)_{n_{1}}\left(a_{2}\right)_{n_{1}}, \ldots,\left(a_{p}\right)_{n_{1}}}{\left(b_{1}\right)_{n_{1}}\left(b_{2}\right)_{n_{1}}, \ldots,\left(b_{q}\right)_{n_{1}}} \frac{1}{n_{1} !} \times \\
\sum_{n_{2}=0}^{y} \frac{\left(a_{1}^{\prime} ; Y\right)_{n_{2}}\left(a_{2}^{\prime}\right)_{n_{2}}, \ldots,\left(a^{\prime}{ }^{\prime}\right)_{n_{2}}}{\left.\left(b_{1}^{\prime}\right)_{n_{2}}\left(b_{2}^{\prime}\right)_{n_{2}}, \ldots, b^{\prime} q^{\prime}\right)_{n_{2}}} \frac{1}{n_{2} !}
\end{array}\right\} \\
& \Gamma\left(1+\omega+\omega^{\prime}-\alpha-\eta+n_{1}\right) \Gamma\left(1+\omega+v^{\prime}-\eta-\alpha+n_{1}\right) \\
& \frac{\Gamma\left(1-v-\alpha+n_{1}\right)}{\Gamma\left(1-\alpha+n_{1}\right) \Gamma\left(1+\omega+\omega^{\prime}+v^{\prime}-\alpha-\eta+n_{1}\right)} x^{\alpha-\omega-\omega^{\prime}+\eta-n_{1}-1} \\
& \Gamma\left(1+\varphi+\varphi^{\prime}-\beta-\tau+n_{2}\right) \Gamma\left(1+\varphi+\delta^{\prime}-\beta-\tau+n_{2}\right) \\
& \frac{\Gamma\left(1-\delta-\beta+n_{2}\right)}{\Gamma\left(1-\beta+n_{2}\right) \Gamma\left(1+\varphi+\varphi^{\prime}+\delta^{\prime}-\beta-\tau+n_{2}\right)} x^{\beta-\varphi-\varphi^{\prime}+\tau-n_{2}-1} \\
& \Gamma\left(1+\varphi-\delta-\beta+n_{2}\right) \\
& \int_{0}^{\infty} \int_{0}^{\infty} e^{-z w-r k} z^{i+n_{1}-1} r^{o+n_{2}-1} d z d r .
\end{aligned}
$$

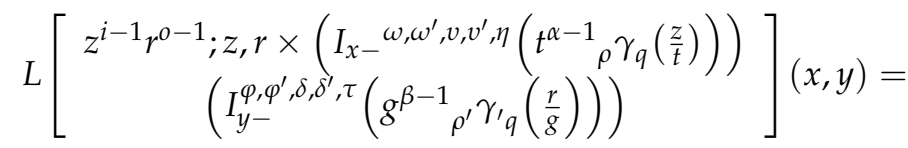

$$
\begin{aligned}
& \left\{\begin{array}{c}
\sum_{n_{1}=0}^{x} \frac{\left(a_{1} ; X\right)_{n_{1}}\left(a_{2}\right)_{n_{1}}, \ldots,\left(a_{p}\right)_{n_{1}}}{\left(b_{1}\right)_{n_{1}}\left(b_{2}\right)_{n_{1}}, \ldots,\left(b_{q}\right)_{n_{1}}} \frac{1}{n_{1} !} \times \\
\sum_{n_{2}=0}^{y} \frac{\left(a_{1}^{\prime} ; Y\right)_{n_{2}}\left(a_{2}^{\prime}\right)_{n_{2}}, \ldots,\left(a^{\prime}{ }^{\prime}\right)_{n_{2}}}{\left(b_{1}^{\prime}\right)_{n_{2}}{ }^{\prime}\left(b_{2}^{\prime}\right)_{n_{2}}, \ldots,\left(b^{\prime} q^{\prime}\right)_{n_{2}}} \frac{1}{n_{2} !}
\end{array}\right\} \\
& \Gamma\left(1+\omega+\omega^{\prime}-\alpha-\eta+n_{1}\right) \Gamma\left(1+\omega+v^{\prime}-\eta-\alpha+n_{1}\right) \\
& \frac{\Gamma\left(1-v-\alpha+n_{1}\right)}{\Gamma\left(1-\alpha+n_{1}\right) \Gamma\left(1+\omega+\omega^{\prime}+v^{\prime}-\alpha-\eta+n_{1}\right)} x^{\alpha-\omega-\omega^{\prime}+\eta-n_{1}-1} \\
& \Gamma\left(1+\omega-v-\alpha+n_{1}\right) \\
& \Gamma\left(1+\varphi+\varphi^{\prime}-\beta-\tau+n_{2}\right) \Gamma\left(1+\varphi+\delta^{\prime}-\beta-\tau+n_{2}\right) \\
& \begin{array}{c}
\Gamma\left(1-\delta-\beta+n_{2}\right) \\
\Gamma\left(1-\beta+n_{2}\right) \Gamma\left(1+\varphi+\varphi^{\prime}+\delta^{\prime}-\beta-\tau+n_{2}\right) \\
\Gamma\left(1+\varphi-\delta-\beta+n_{2}\right)
\end{array} x^{\beta-\varphi-\varphi^{\prime}+\tau-n_{2}-1} \\
& \frac{(i)_{n_{1}} \Gamma(i)}{w^{i}} \frac{(o)_{n_{2}} \Gamma(o)}{k^{o}} .
\end{aligned}
$$

Hence, with some calculation, we are able to obtain the correct result. For the second assertion, take an incomplete hypergeometric function (12) and proceed the same way.

\section{Special Cases:}

We provide some special cases by selecting appropriate values for the variables $\omega, \omega^{\prime}, v, v^{\prime}$ and $\eta ; \varphi, \varphi^{\prime} \delta, \delta^{\prime}$ and $\tau$. If we put $\omega=\omega+v, \omega^{\prime}=v^{\prime}=0, v=-\eta$; $\varphi=\varphi+\delta, \varphi^{\prime}=\delta^{\prime}=0, \delta=-\tau$ into Theorems 1 and 2, respectively, then we acquire interesting results concerning the Saigo fractional integral operators that are presented by the following corollaries.

\subsection{Corollary 1}

Assume $\omega, v, \eta ; \varphi, \delta, \tau \in C$ such that $(\Re(\eta)>0, \Re(\tau)>0)$ if the condition $\Re(\eta)>\max [0, \Re(\eta-\omega),(v-\omega)]$ and $\Re(\tau)>\max [0, \Re(\tau-\varphi),(\delta-\varphi)]$ holds. Thus, 
the left-sided Saigo-Maeda operator of integration for an incomplete hypergeometric function is presented as:

$$
\begin{aligned}
& L\left[\begin{array}{c}
z^{i-1} r^{o-1} ; z, r \times\left(I_{x+}{ }^{\omega, v, \eta}\left(t^{\alpha-1}{ }_{\rho} \gamma_{q}(z t)\right)\right) \\
\left(I_{y+}^{\varphi, \delta, \tau}\left(G^{\beta-1}{ }_{\rho^{\prime}} \gamma_{q_{q}}(r g)\right)\right)
\end{array}\right](x, y)= \\
& \frac{1}{w^{i} k^{0}} x^{\alpha-v-1} x^{\beta-\delta-1} \frac{\Gamma(i) \Gamma(o) \Gamma(\alpha) \Gamma(\beta) \Gamma(\alpha+\eta-v) \Gamma(\beta+\tau-\delta)}{\Gamma(\alpha-v) \Gamma(\beta-\delta) \Gamma(\alpha+\eta+\omega) \Gamma(\beta+\tau+\varphi)}
\end{aligned}
$$

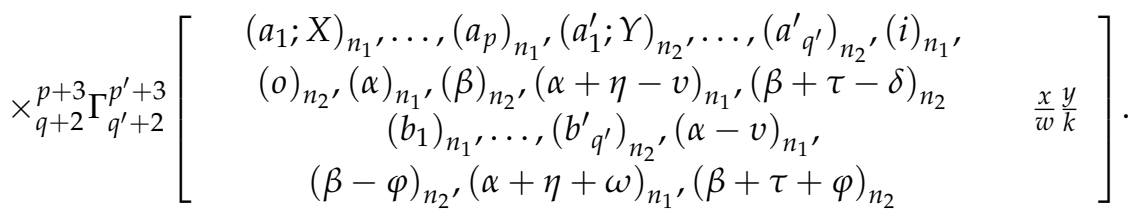

and

$$
\begin{aligned}
& L\left[\begin{array}{c}
z^{i-1} r^{o-1} ; z, r \times\left(I_{x+}{ }^{\omega, v, \eta}\left(t^{\alpha-1}{ }_{\rho} \gamma_{q}(z t)\right)\right) \\
\left(I_{y+}^{\varphi, \delta, \tau}\left(G^{\beta-1}{ }_{\rho^{\prime}} \gamma_{q}(r g)\right)\right)
\end{array}\right](x, y)= \\
& \frac{1}{w^{i} k^{0}} x^{\alpha-v-1} x^{\beta-\delta-1} \frac{\Gamma(i) \Gamma(o) \Gamma(\alpha) \Gamma(\beta) \Gamma(\alpha+\eta-v) \Gamma(\beta+\tau-\delta)}{\Gamma(\alpha-v) \Gamma(\beta-\delta) \Gamma(\alpha+\eta+\omega) \Gamma(\beta+\tau+\varphi)}
\end{aligned}
$$

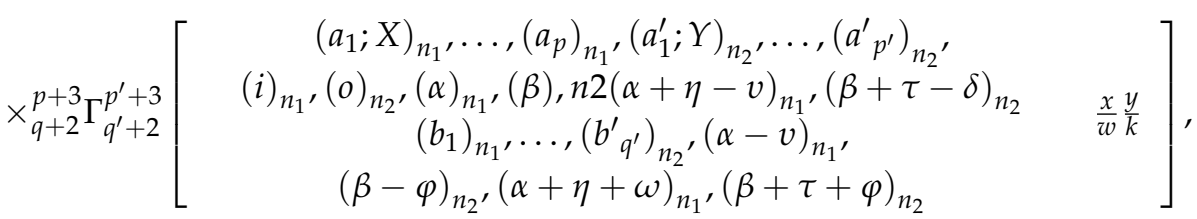

For the second assertion, take the incomplete hypergeometric function (12) and proceed in the same way.

\subsection{Corollary 2}

Assume $\omega, v, \eta ; \varphi, \delta, \tau \in \mathrm{C}$ and $\left|\frac{1}{t}\right| \leq 1,\left|\frac{1}{g}\right| \leq 1$ such that $(\Re(\eta)>0, \Re(\tau)>0)$; if the condition $\Re(\eta)>-\min [0, \Re(\eta-\omega),(v-\omega)]$ and $\Re(\tau)>-\min [0, \Re(\tau-\varphi),(\delta-\varphi)]$ is satisfied, then the left-sided Saigo-Maeda operator of integration of an incomplete hypergeometric function is given by

$$
\begin{aligned}
& L\left[\begin{array}{c}
z^{i-1} r^{o-1} ; z, r \times\left(I_{x-}{ }^{\omega, v, \eta}\left(t^{\alpha-1}{ }_{\rho} \gamma_{q}(z t)\right)\right) \\
\left(I_{y-}^{\varphi, \delta, \tau}\left(G^{\beta-1}{ }_{\rho^{\prime}} \gamma_{\gamma_{q}}(r g)\right)\right)
\end{array}\right](x, y)=
\end{aligned}
$$

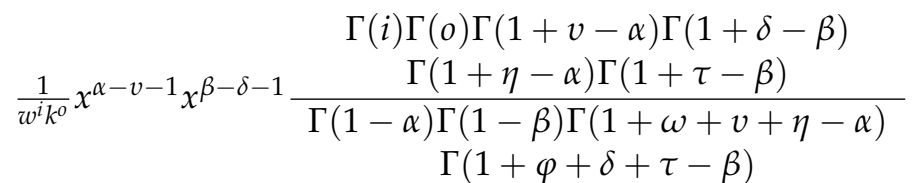

$$
\begin{aligned}
& \times{ }_{q+2}^{p+3} \Gamma_{q^{\prime}+2}^{p^{\prime}+3}\left[\begin{array}{cc}
\left(a_{1} ; X\right)_{n_{1}, \ldots,}\left(a_{p}\right)_{n_{1}}\left(a_{1}^{\prime} ; Y\right)_{n_{2}}, \ldots,\left(a_{q^{\prime}}^{\prime}\right)_{n_{2}{ }^{\prime}} & \\
(i)_{n_{1}}(o)_{n_{2}}(1+v-\alpha)_{n_{1}}, \ldots,(1-\delta-\beta)_{n_{2}} & \frac{1}{x w} \frac{1}{y k} \\
\left(b_{1}\right)_{n_{1}}, \ldots,\left(b_{q^{\prime}}^{\prime}\right)_{n_{2}}(1-\alpha)_{n_{1}}, \ldots, & \\
(1+\varphi-\delta-\beta)_{n_{2}} &
\end{array}\right]
\end{aligned}
$$


and

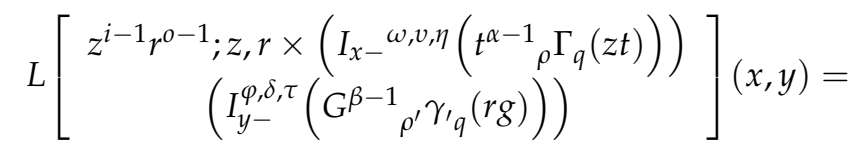

$$
\begin{aligned}
& \Gamma(i) \Gamma(o) \Gamma(1+v-\alpha) \Gamma(1+\delta-\beta) \\
& \frac{1}{w^{i} k^{0}} x^{\alpha-v-1} x^{\beta-\delta-1} \frac{\Gamma(1+\eta-\alpha) \Gamma(1+\tau-\beta)}{\Gamma(1-\alpha) \Gamma(1-\beta) \Gamma(1+\omega+v+\eta-\alpha)} \\
& \Gamma(1+\varphi+\delta+\tau-\beta)
\end{aligned}
$$

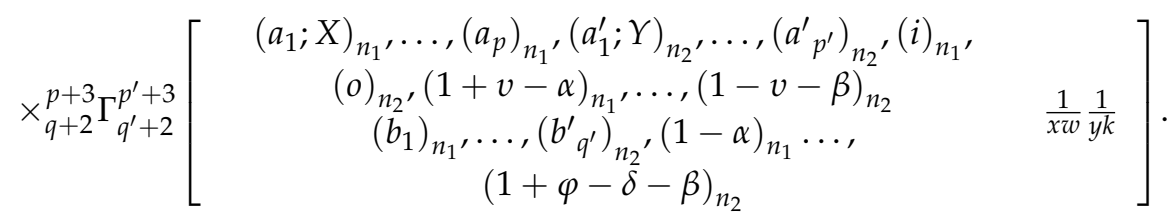

For the second assertion, take an incomplete hypergeometric function (12) and proceed the same way.

Remark: We obtained a two-dimensional Laplace transform that involved the twodimensional MSM integral operator. The two-dimensional Saigo-Maeda integral operator is the unique aspect of our main finding. Furthermore $v=-\omega ; \delta=-\varphi$. These Saigo fractional integrals are in agreement with Riemann-Liouville integral, and if we use the parameters $v=0 ; \delta=0$, then the Saigo fractional integral operator becomes an ErdélyiKober-type fractional integral operator.

\section{Conclusions}

We established a composite result using the Laplace transform, which helps to extend the generalized incomplete hypergeometric function. As with the assistance of the famous MSM fractional differential operator, it seems that Laplace has a strong effect on the behavior of the incomplete hypergeometric function, which makes it more generalized. Furthermore, after using the two-dimensional Laplace with the two-dimensional MSM integral operator with the two-dimensional incomplete hypergeometric function, this work enters a very new domain that has not been seen before. By using the appropriate techniques with the parameters, we were able to convert the MSM differential and integration operator into the Saigo differential and integral operator in corollaries. As such, the results of our findings lead us to applications in various fields.

Author Contributions: Data curation, Y.K.; Formal analysis, A.K.; Funding acquisition, M.S.; Investigation, A.A. All authors have read and agreed to the published version of the manuscript.

Funding: Deanship of Scientific Research, University of Hafr Al Batin, Research group project no. (G-108-2020).

Institutional Review Board Statement: Not applicable.

Informed Consent Statement: Not applicable.

Data Availability Statement: Not applicable.

Acknowledgments: The authors extend their appreciation to the Deanship of Scientific Research, University of Hafr Al Batin for funding this work through research group project no. (G-108-2020).

Conflicts of Interest: The authors declare no conflict of interest.

\section{References}

1. Khan, A.; Manzoor, T.; Kahsay, H.A.; Wubneh, K.G. Laplace Operator with Caputo-Type Marichev-Saigo-Maeda Fractional Differential Operator of Extended Mittag-Leffler Function. Discret. Dyn. Nat. Soc. 2021, 2021, 5587616. [CrossRef]

2. Manzoor, T.; Khan, A.; Wubneh, K.G.; Kahsay, H.A. Beta Operator with Caputo Marichev-Saigo-Maeda Fractional Differential Operator of Extended Mittag-Leffler Function. Adv. Math. Phys. 2021, 2021, 5560543. [CrossRef] 
3. Bansal, M.K.; Kumar, D.; Harjule, P.; Singh, J. Fractional Kinetic Equations Associated with Incomplete I-Functions. Fractal Fract. 2020, 4, 19. [CrossRef]

4. Chauhan, R.; Kumar, N.; Aggarwal, S. Dualities between Laplace-Carson Transform and Some useful integration. IJITEE 2019, 8, 2278-3075.

5. Agarwal, P.; Qi, F.; Chand, M.; Jain, S. Certain integrals involving the generalized hypergeometric function and the Laguerre polynomials. J. Comput. Appl. Math. 2017, 313, 307-317. [CrossRef]

6. Agarwal, P.; Qi, F.; Chand, M.; Singh, G. Some fractional differential equations involving generalized hypergeometric functions. J. Appl. Anal. 2019, 25, 37-44. [CrossRef]

7. Saxena, R.K.; Ram, J.; Kumar, D. On the two-dimensional Saigo-Maeda fractional calculus is associated with two-dimensional aleph transform. Le Mat. 2013, LXVIII-Fasc. II, 267-281.

8. Shaeel, M.; Khan, A.; Hasnain, S.A. Laplace and Inverse Laplaced Transform and Generalized Incomplete Hypergeometric Functions. Pak. J. Stat. 2021, 34, 417-428.

9. Srivastava, H.M.; Saxena, R. Operators of fractional integration and their applications. Appl. Math. Comput. 2001, 118, 1-52. [CrossRef]

10. Rainville, E.D. Special Functions; The Macmillan Company: New York, NY, USA, 1971.

11. Srivastava, H.M.; Chaudhry, M.A.; Agarwal, R.P. The incomplete Pochhammer symbols and their applications to hypergeometric and related functions. Integral Transform. Spec. Funct. 2012, 23, 659-683. [CrossRef]

12. Saigo, M.; Maeda, N. More generalization of fractional calculus. In Transform Methods E Special Functions, Varna'96 (Proc. Second Internat. Workshop); Rusev, P., Dimovski, I., Kiryakova, V., Eds.; Science Culture Technology Publishing: Singapore, 1998; pp. 386-400.

13. Nadir, A.; Khan, A. Marichev-Saigo-Maeda Differential operator and Generalized Incomplete hypergeometric functions. Punjab Univ. J. Math. 2018, 50, 123-129.

14. Saigo, M. A remark on integration operators involving the Gauss hypergeometric functions. Math. Rep. Coll. Gen. Ed. Kyushu Univ. 1978, 11, 135-143. 